(to be submitted to J. Chem. Phys. )

\title{
Efficient moves for global geometry optimization methods and their application to binary systems
}

\author{
Michael Sicher, ${ }^{1}$ Stephan Mohr, ${ }^{1}$ and Stefan Goedecker ${ }^{1}$ \\ Department of Physics, Universität Basel, Klingelbergstr. 82, 4056 Basel, \\ Switzerland
}

(Dated: 8 August 2018)

We show that molecular dynamics based moves in the Minima Hopping (MH) method are more efficient than saddle point crossing moves which select the lowest possible saddle point. For binary systems we incorporate identity exchange moves in a way that allows to avoid the generation of high energy configurations. Using this modified Minima Hopping method we reexamine the binary Lennard Jones (BLJ) benchmark system with up to 100 atoms and we find a large number of new putative global minima structures.

PACS numbers: 36.40.Mr, 61.46.Bc

\section{INTRODUCTION}

In a global geometry optimization one has to search over many local minima until one finds the global minimum. Moves 1,2 are necessary to jump from the catchment basin of the current local minimum into another catchment basin. The types of moves that are chosen for this hopping from one catchment basin into another has an important effect on the efficiency of the method. The majority of the moves used by researches in this field, fall into the following five categories.

- Random Moves: The atoms are displaced randomly from the current positions. If the amplitude of the random displacement is large enough, the system will relax into another local minimum during a standard local geometry optimization. Random moves are widely used both within random search methods ${ }^{3,4}$ and within basin hopping ${ }^{5}$.

- Molecular dynamics moves: One of the oldest global optimization methods, simulated anneal$\operatorname{ing}^{6}$ is based on a modified molecular dynamics scheme where the temperature is lowered continuously. Molecular dynamics is also used as a move in the Minima Hopping $\operatorname{method}^{7}$ as well as in various other schemes $\frac{8,9}{}$.

- Library based moves: For systems such as proteins, where one knows in advance which kind of moves are important, non-physical moves, based on a library of moves can be used ${ }^{10}$.

- Transition state search based moves: Starting from a given local minimum one searches for a neighbouring saddle point. The system is then moved over the saddle point and afterwards relaxed into the local minimum across this saddle point. This kind of move is used in the ART method ${ }^{11}$, which is primarily a method to explore potential energy landscapes, but which will also visit the global minimum if run long enough. Saddle points are also determined in the TAD method ${ }^{12}$ which allows to determine rates of rare events.
- Genetic algorithms ${ }^{13}$ use mutation and crossover operations which can also be considered as moves. These moves are typically strongly system dependent and the moves for clusters 14 are for instance different to the moves used for crystal structure prediction 15 .

The density of configurations is increasing exponentially with increasing energy. It is therefore important to search only over an energy interval above (and including) the global minimum which is not too large. Otherwise the number of configurations in this interval is so large that one will miss most likely the global minimum. This means that one should use moves that will never or only rarely lead into high energy structures. If moves with this property are not used, the majority of the configurations has to be discarded in an acceptance/rejectance step which can be found in most global optimization algorithms. A large rejectance ratio is however also highly inefficient. If one considers moves that lead from one minimum into a neighbouring one, it has been shown that molecular dynamics based moves are very efficient ${ }^{16}$. Since a molecular dynamics trajectory has a fixed and limited kinetic energy it follows from energy conservation that it cannot go over barriers that are higher than this kinetic energy. Since the Bell Evans Polanyi principle ${ }^{17}$ tells us that minima behind low energy barriers are on average also low in energy, molecular dynamics based moves do not lead into high energy structures if their kinetic energy is chosen such that they can only overcome low energy barriers.

In this paper we will first compare two classes of moves that both are able to find low energy escape paths from a current minimum, namely molecular dynamics based moves with moves that are saddle point based. In the second part of the paper we will discuss moves for binary systems. We will in particular discuss under which circumstances moves that exchange the identity of two atoms are efficient. In the third part we will apply our resulting global optimization scheme to the benchmark set of binary Lennard Jones (BLJ) clusters $^{18}$ and show that many global minimum structures had been overlooked in previous studies. The LJ potential poses the same kind of problems for global geometry optimization methods as 
other more realistic potentials $\frac{19}{}$ and the efficiency of an algorithm for an LJ system is therefore indicative of the success for other potentials.

\section{SADDLE POINT ESCAPE MOVES VERSUS MOLECULAR DYNAMICS ESCAPE MOVES}

We will now compare the efficiency of various moves within the Minima Hopping method. The original MH method consists of a sequence of short molecular dynamics trajectories followed by local geometry optimizations ${ }^{7}$. The molecular dynamics trajectory allows to cross barriers to hop from one catchment basin into another and the local geometry optimization will then bring the system to the bottom of the catchment basin.

The other kind of moves are based on saddle point searches. Starting from a local minimum, the system is propagated towards a saddle point. After reaching such a transition state and barely crossing it, a local geometry optimization brings the system again down to a new minimum. We use the dimer method 20 with a few modifications to search the saddle points and to identify the transition states in this modified version of the $\mathrm{MH}$ method.

The dimer consists of two points $\mathbf{R}_{1}$ and $\mathbf{R}_{2}$ in the high dimensional space in which one wants to locate the transition state. If the dimer midpoint is labelled as $\mathbf{R}$, then these two points are formed according to $\mathbf{R}_{1}=\mathbf{R}+$ $\Delta R \hat{\mathbf{N}}$ and $\mathbf{R}_{2}=\mathbf{R}-\Delta R \hat{\mathbf{N}}$, where $\hat{\mathbf{N}}$ is the normalized dimer direction. The dimer method consists of basically two steps. In the first one, the dimer is rotated into a position which gives a small torsional force and which aligns it with an eigenmode of the Hessian. This torsional force is given by $\mathbf{F}^{\perp}=\left(\mathbf{F}_{1}-\mathbf{F}_{\mathbf{2}}\right)-\left\langle\mathbf{F}_{\mathbf{1}}-\mathbf{F}_{\mathbf{2}} \mid \hat{\mathbf{N}}\right\rangle \hat{\mathbf{N}}$, with $\mathbf{F}_{1}$ and $\mathbf{F}_{2}$ being the forces at $\mathbf{R}_{1}$ and $\mathbf{R}_{2}$, respectively. Since the dimer midpoint remains constant during the rotation, the force acting on $\mathbf{R}_{2}$ can be approximated by $\mathbf{F}_{2}=$ $2\left(\mathbf{F}-\mathbf{F}_{1}\right)$ in order to reduce the total number of force evaluations, $\mathbf{F}$ denoting the force at the dimer midpoint. In the second step the dimer is translated along $\mathbf{F}_{e f f}$, where this modified force is given by $\mathbf{F}_{e f f}=-\langle\mathbf{F} \mid \hat{\mathbf{N}}\rangle \hat{\mathbf{N}}$ if the curvature along the dimer axis is negative and by $\mathbf{F}_{e f f}=\mathbf{F}-10\langle\mathbf{F} \mid \hat{\mathbf{N}}\rangle \hat{\mathbf{N}}$ otherwise.

Whereas the translation is always done in a straightforward way, there exist several different ways how the rotation can be carried out. Since the rotation part requires considerably more force evaluations than the translation part, choosing a good rotation method is important. Most methods have the tendency to align the dimer along the lowest curvature mode. This is due to the fact that only the lowest curvature mode is a minimum with respect to the curvature; all other low curvature modes are saddle points. Whereas this circumstance does not cause any problem if one is interested in finding only one saddle point leading out of a given minimum, it is a severe shortcoming in our case. Due to this behaviour it is very likely that the dimer aligns itself with the lowest curvature mode after a few iterations even if it was initially aligned along another than the lowest mode and, as a consequence, several searches will lead to the same saddle point, even if they were originally started in different orthogonal directions. This is a serious problem since we are interested in finding many different saddle points leading out of a given minimum and need therefore a rotation method which keeps the dimer on the initially selected mode, thus leading to distinct saddle points.

One possibility that was already mentioned in the original paper ${ }^{20}$ is to impose the restriction that the dimer is orthogonal to all previous dimer directions at the minimum until the dimer is aligned along the lowest mode itself - which will happen as we approach the saddle point - since then there is no more tendency for the dimer to switch to another mode. This procedure requires however the knowledge of all lower lying modes if one is interested to follow a higher mode. Since in our new method we are interested in finding systematically all low lying saddle points around a local minimum this condition is automatically fulfilled. However, we also develop another method where it is necessary to directly follow a higher mode and this orthogonalization procedure is as a consequence not suited, and we therefore will look for another way to stay on the initially selected mode.

Tests with several rotation methods show that using Direct Inversion in Iterative Subspace (DIIS) 21 is most suitable for our purpose, since DIIS has the tendency to catch the nearest lying stationary point, regardless of whether it is a minimum, maximum or saddle point. This allows us to stay on the initially selected mode with high reliability. Approximating the error vectors by $-\alpha \mathbf{F}^{\perp}$, where $\alpha$ is a constant, we move $\mathbf{R}_{1}$ according to the standard DIIS procedure with the modification that $\mathbf{R}_{1}$ has to be adjusted after each step to retain the fixed dimer separation. However, it turns out that it is for reasons of stability not good to stay on the initially selected mode at any cost, but to follow the lowest mode at some point instead. This can easily be achieved by abandoning the DIIS rotation and using the Lanczos method thenceforth. This switch to the Lanczos method was done as soon as the second derivative of the energy with respect to the number of iterations became negative.

In the present implementation of our saddle point searches we put the focus on reliability and not on speed, since we are only interested in understanding the principle of the various types of moves. Therefore about 1000 force evaluations are required if we want to have a success rate of some 99 percent. Further tuning might still bring down the number of force evaluations, but it seems unlikely that it can be reduced by one order of magnitude, which would be necessary to compete with molecular dynamics based moves. So it is clear that saddle point based moves are only of interest in practice if the global minimum can be found much faster with respect to the number of minima that have to be visited until the global one is found. 
With the exception of the moves, whose details will be explained below, the standard $\mathrm{MH}$ algorithm was used, i.e. new minima are accepted if they are not higher than $E_{\text {diff }}$ in energy and the value of $E_{\text {diff }}$ is adjusted by a feedback mechanism such that on average half of the new configurations are accepted.

We use the Lennard-Jones clusters $L J_{55}$ and $L J_{38}$ as test systems because they behave very differently. The $L J_{55}$ is a structure seeker for which it is very easy to find the global minimum. $L J_{38}$ on the other hand is a two funnel system for which it is surprisingly difficult to find its global minimum in view of its small size. 100 global optimization runs are done in all cases to get well defined average values.

\section{A. Crossing the lowest barrier}

The first saddle point based type of move is conceptually simple. According to the Bell Evans Polanyi principle, the ideal move would be a move which escapes from the current catchment basin by going over the lowest barrier. There are however two problems using this type of move. In case a minimum is visited a second time, the sequence of minima that are visited would repeat itself ad infinitum and no new minima would be visited. The system would not be ergodic in a certain sense. This problem can easily be overcome by a small modification of the method. If a minimum is visited for the first time one escapes over the lowest barrier, if it is visited a second time one escapes over the second lowest barrier and so on. The second problem is that such a type of move would be incredibly expensive numerically. Doing a one sided search for a single saddle point requires typically already a few hundred force evaluations and exploring more or less all the saddle points around a local minimum to find out which is the lowest one is even much more expensive. At this stage we are however only interested in understanding the efficiency of a certain type of move and we will for the moment not care about the cost of a single move. We will measure the efficiency of the moves by counting how many local minima will be visited on average in this modified saddle point search based version of the Minima Hopping algorithm before the global minimum is found and we will ignore the fact that the CPU time can be very long due to the cost of the moves. In our implementation of this method we perform 50 saddle point searches starting from the current local minimum. Out of these we choose the one exhibiting the lowest barrier. Since the saddle point searches sometimes give saddle points which are not connected to the initial minimum (meaning that a local geometry optimization starting at that saddle point would not lead back to the initial minimum), these barriers may be meaningless. However, we do not care about this fact and simply choose that saddle point with the lowest value.

An overview of the performance with this method, which we denote as lowest barrier (LB), is given in Ta- ble I. We compare the performance of the LB method to that of the standard molecular dynamics (MD) version and to that of the saddle point based lowest mode (LM) method which will be discussed in section ПB. As usual $^{22}$ we start our molecular dynamics trajectory in a soft direction, i.e. in a direction with low curvature in order to overcome a low barrier with a small number of molecular dynamics steps. This direction should however not exactly be identical to the direction of the lowest curvature, i.e. the lowest eigenvector of the Hessian matrix, because we would again loose ergodicity in this way. We need enough randomness in the initial direction of the velocity vector to be able to jump into different catchment basins when we escape repeatedly from a certain minimum.

As one sees, the LB method is somewhat more efficient than MD in terms of the number of distinct local minima that are visited before finding the global minimum for $L J_{38}$. In terms of the total number of minima the MD based escapes are however more efficient. This comes from the fact that the MD based escapes are more ergodic than the LB based escapes and repeated visits of the same minimum are therefore less likely. Fig. 1 shows that a MD trajectory has a large choice for crossing very low barriers, i.e. there are many low lying saddle point around a local minimum. In the case of $L J_{55}$ the MD based escapes are more efficient according to both criteria. The surprising result that $\mathrm{MD}$ is more efficient than LB comes from the fact that the molecular dynamics trajectory can cross several barriers whereas in the LB based moves one crosses by definition only one barrier. In the case of the $L J_{55}$ cluster crossings of several barriers are frequently encountered since the whole energy landscape is strongly 'tilted' in the direction of the global minimum.

\begin{tabular}{lrrrrr}
\hline & \multicolumn{2}{c}{$L J_{38}$} & & \multicolumn{2}{c}{$L J_{55}$} \\
\cline { 1 - 3 } & total & different & & total & different \\
minima & minima & & minima & minima \\
MD & 2703.6 & 523.9 & & 415.5 & 91.9 \\
SP LB & 1030.1 & 297.4 & & 92.3 & 28.4 \\
\hline
\end{tabular}

TABLE I. Comparison of the performance of all three Minima Hopping versions for both $L J_{38}$ and $L J_{55}$ clusters. "total minima" are the total number of visited minima per run, "different minima" indicates how many among them are different. "SP LM" means the saddle point version that follows the lowest mode, "SP LB" the one that crosses the lowest barrier. The data is based on 100 runs for each version.

\section{B. Following the lowest mode}

Even though the results for the LB case are already discouraging, we present a second scheme which is more realistic since it does not require to find all the barriers around a local minimum to make a single move. In this scheme we exploit the fact that there is a correla- 
tion between the curvature of the direction into which we start our saddle point search and the height of the saddle point found. This correlation is shown in Fig. 1 and it is the same correlation that is also exploited when we start our molecular dynamics trajectories in soft directions. In this scheme, which we denote by lowest mode (LM), we search for the saddle point in the softest direction if the minimum is visited for the first time, in the second softest direction if it is visited for the second time and so on. Using DIIS for the dimer rotation ensures that these searches will lead to distinct saddle points with high probability. If we find a saddle point we will move the system over this saddle point and perform a local geometry optimization. Each mode gives us two degenerate directions, namely the direction of the positive and negative eigenvector of this mode, and we can therefore perform two saddle point searches for each mode.

The results in Table I show that this approach is in all cases much less efficient than the molecular dynamics based moves. It is due to the fact that even if we start our saddle point search in a soft direction we can frequently obtain very high saddle points, whereas in the molecular dynamics based moves energy conservation will prevent the crossing of such high barriers. The energy of the molecular dynamics trajectory is usually much larger than needed to cross a barrier, and one would therefore not expect that there is a correlation between the energy of the trajectory crossing from one catchment basin into another one and the height of the saddle point that connects the minima of the two catchment basins. However, we found that such a correlation does indeed exist, as shown in Fig 2 .

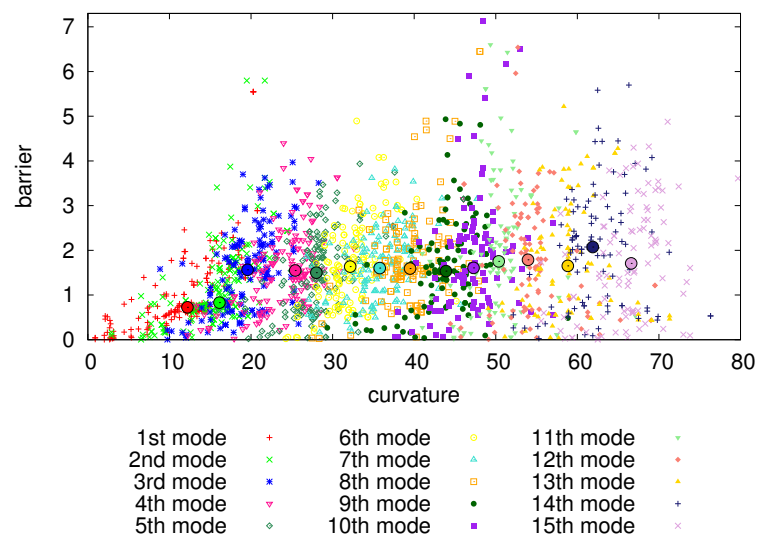

FIG. 1. A plot of the barrier heights as a function of the curvature along the direction in which the saddle point search was started. In addition the numbers of the modes along which the search was started are distinguished by colours. The median value for each mode (median value of the curvature as well as the median value of the barrier) is plotted with a large dot. One can see that it is very unlikely to find high barriers along the softest (lowest curvature) directions. Along the stiffer (higher curvature) directions one finds increasingly higher barriers but in addition there exist also low barriers.

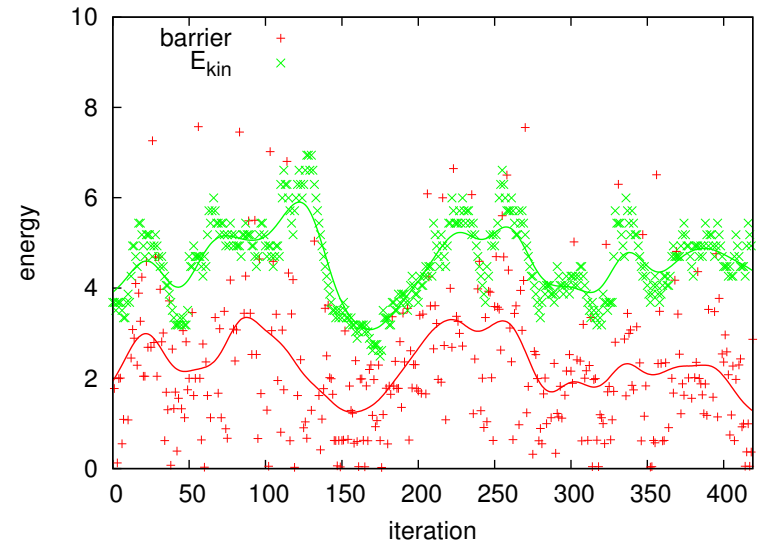

FIG. 2. The correlation between barrier heights and kinetic energy during a MD Minima Hopping run. The kinetic energy is scaled down by a factor of three for a better visualization. The straight lines are convolutions of the data with a Gaussian. There is a clear correlation between the barrier height and the kinetic energy, even if the kinetic energy is much higher than the barriers.

\section{MOVES FOR BINARY SYSTEMS}

In the case of binary systems one might think that molecular dynamics based moves are inefficient since atoms of a certain type will only move by some slow diffusive motion to their right place. A process which can bring atoms potentially faster to their right place is an identity exchange where the identity of two atoms that are possibly far apart is exchanged. In a binary LennardJones (BLJ) cluster the identities will be denoted by A and B. On the other hand we know that the efficiency of the molecular dynamics based moves in Minima Hopping is due to the fact that high energy configurations are rarely visited which leads to small values of $E_{\text {diff }}$. Table II shows these values for several BLJ systems which have different size-mismatch values $\sigma$. The $E_{\text {diff }}$ value is always the value which gives on average an acceptance ratio of 0.5 in the standard $M D$ based version of $M H$. $\sigma=\frac{\sigma_{B B}}{\sigma_{A A}}$ is the size of the larger type-B atom in a BLJcluster where the smaller type-A atoms are chosen to have size 1 . The interaction potential is then given by

$$
E=4 \sum_{i<j} \epsilon_{\alpha \beta}\left[\left(\frac{\sigma_{\alpha \beta}}{r_{i j}}\right)^{12}-\left(\frac{\sigma_{\alpha \beta}}{r_{i j}}\right)^{6}\right]
$$

where $\alpha$ and $\beta$ are the types of atoms $i$ and $j . \sqrt[6]{2} \sigma_{\alpha \beta}$ is the equilibrium pair separation and $\epsilon_{\alpha \beta}$ is the well depth of the pair potential from atoms $i$ and $j$. We set $\epsilon_{A A}=\epsilon_{B B}=\epsilon_{A B}=\epsilon=1$ and $\sigma_{A B}=\frac{\sigma_{A A}+\sigma_{B B}}{2}$. With these settings, the only free parameter besides the number of type-A atoms $N_{A}$ is $\sigma$ which is chosen to be $\sigma \in\{1,1.05, \ldots, 1.3\}$.

TableII also shows the acceptance ratio for identity exchange moves followed by a local geometry optimization, 


\begin{tabular}{cccc}
\hline$\sigma$ & system & $E_{\text {diff }}$ & acc./rej. \\
\hline 1.05 & $A_{19} B_{26}$ & 0.44 & 1.50 \\
1.15 & $A_{31} B_{63}$ & 0.66 & 0.14 \\
1.20 & $A_{33} B_{63}$ & 0.37 & 0.03 \\
1.25 & $A_{42} B_{58}$ & 0.46 & 0.01 \\
\hline
\end{tabular}

TABLE II. acceptance/rejectance ratio for identity exchange moves with fixed $E_{\text {diff }}$.

if the $E_{\text {diff }}$ of the MD move for the same system is used. One can see that these acceptance ratios get smaller and smaller with increasing $\sigma$. This means that in most cases exchanging two atom types will lead to rather high energy configurations and is hence less efficient than MD moves. In nature real atoms do not only differ by size (e.g. covalent radius) but also by their electronic properties. Exchange moves are therefore expected to be efficient only if the atoms are very similar in every respect. If the atoms are very different there is actually a strong driving force present in the MD moves to put the different types of atoms at the right positions. If the global minimum structure is for instance a core-shell structure we obtain a core-shell like structure starting from a random position already after some 100-1000 MD moves (see Fig 3). Atomic identity exchange moves are therefore not only not necessary, but would even be counterproductive.

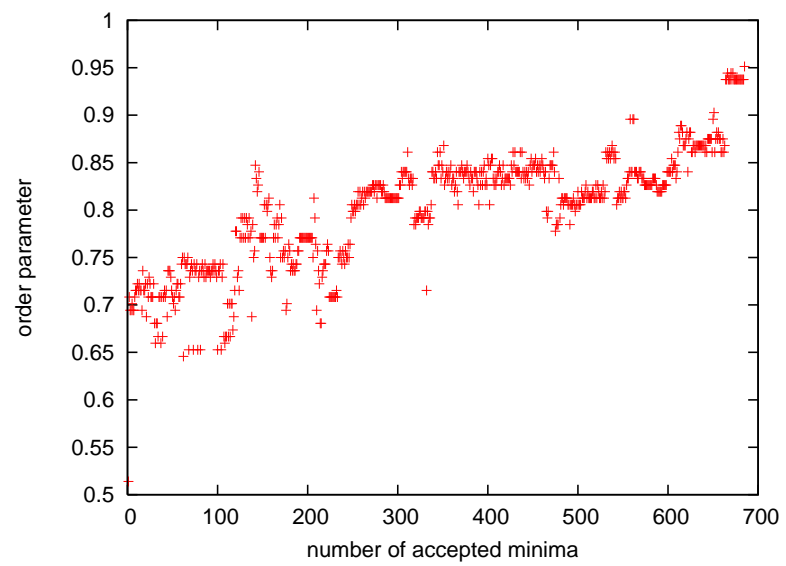

FIG. 3. Figure shows how fast a core shell structure of a BLJcluster is formed if one starts from a random distribution of atoms of type $\mathrm{A}$ and $\mathrm{B}$. The order parameter is defined as the fraction of atoms which are in the core. An order parameter of 1 corresponds thus to the global minimum structure of a particular system. The evolution towards the core-shell structure is plotted only until the order parameter reaches the value 0.95 where the core-shell structure is already clearly visible. The data is obtained for the system $A_{39} B_{12}$ with $\sigma=1.1$

For systems with two similar types of atoms (i.e. small $\sigma$ for BLJ-systems) identity exchange moves can however reduce the average search time for the global minimum. Due to the significantly lower acceptance ratio at constant $E_{\text {diff }}$, these exchange moves can not be treated on an equal footing as the MD moves. In particular it would be too expensive to do a local geometry optimization after each exchange move. For this reason we have incorporated exchange moves in the following way in our Minima Hopping algorithm: After each MD move we do a number of exchange moves which is roughly equal to the number of force evaluations required in the geometry optimization. If the energy of the unrelaxed configuration resulting from this exchange move increases by less than $E_{\text {relax }}$ with respect to the original configuration it is relaxed and taken as the result of this combined $\mathrm{MD} /$ exchange move. $E_{\text {relax }}$ is the energy that is on average gained by a local geometry optimization starting from a relaxed configuration where the identity of two atoms is exchanged. Hence the relaxed energy of the exchanged configuration will be on average lower then the energy of the original configuration. In this way the exchange moves can help in finding the global minimum even if their acceptance probability is lower than the acceptance probability of the MD moves by a factor which is roughly equal to the number of force evaluations needed by the MD moves.

\section{RESULTS FOR THE BINARY LJ BENCHMARK SYSTEMS}

Finding the global minimum for a binary LennardJones cluster is significantly more difficult than for a mono-atomic Lennard-Jones cluster. In the limit where the two atoms become identical (but are still slightly different) each configuration becomes degenerate with a degeneracy of $\frac{\left(N_{A}+N_{B}\right) !}{N_{A} ! N_{B} !}$, where $N_{A}$ is the number of BLJ atoms of type $\mathrm{A}$ and $N_{B}$ the number BLJ atoms of type B. Such configurations, that can be transformed into each other by identity exchanges of the atom types, are called homotopes 23 .

If the type-A and type- $\mathrm{B}$ atoms have a larger sizemismatch $\sigma$, not all $\frac{\left(N_{A}+N_{B}\right) !}{N_{A} ! N_{B} !}$ homotopes are stable, but nevertheless a smaller number of homotopes exist. The existence of stable homotopes increases the number of local minima of binary systems compared to the monoatomic case. Depending on the system size and composition this number can thus be significantly larger. The difficulty of the global optimization of binary LennardJones systems is also reflected in the data of the Cambridge Cluster database ${ }^{18}$. For mono-atomic LennardJones systems the putative global minima up to a cluster size of 1000 atoms are listed, but for BLJ-systems only up to 100 atoms. Since the putative global minima structures are given for 6 different size ratios $\sigma$ the database contains 600 structures. A first computation of the putative global minima in this database was done by Doye et al ${ }_{25}^{25}$. Andrea Cassioli, Marco Locatelli and Fabio Schoen ${ }^{26}$ reexamined the problem and found nearly 100 new putative global minima for the 600 structures in the database. A few new structures were also found by Pullan27. In spite of the fact that several groups have already reexamined the database we were able to find the 
following 17 structures which are lower in energy than the structures listed in the database (status June 2006):

$$
\begin{aligned}
& \text { - } \sigma=1.30: B L J_{100}, B L J_{99}, B L J_{98}, B L J_{97}, B L J_{96} \\
& \text { - } \sigma=1.25: B L J_{100}, B L J_{99}, B L J_{98}, B L J_{97}, B L J_{96} \\
& \text { - } \sigma=1.20: B L J_{100}, B L J_{97}, B L J_{96}, B L J_{95} \\
& \text { - } \sigma=1.15: B L J_{94}, B L J_{93}, B L J_{92} .
\end{aligned}
$$

The global optimization runs for systems with a size-mismatch ratio of $\sigma \geq 1.2$ were done with the standard MH algorithm ${ }^{7}$. The putative global minima with $\sigma=1.15$ have been found by using the above mentioned identity moves which turned out to be a powerful additional feature to the $\mathrm{MH}$ algorithm for the global optimization of binary systems with comparable atomic sizes.

We did not systematically recalculate the whole Cambridge cluster database. However, a visualization of the putative global minimum structures provided by the database revealed that several structures didn't fit into the "series" under the same $\sigma$ due to too much disorder of the clusters or a still incomplete separation of core and shell. These structures were reexamined and new energetically lower structures, that frequently had also different stoichiometric compositions, were found in many cases.

\section{A. New putative global minima}

We now present the structures corresponding to the new putative global minima. Using the classification criteria of Doye et al $\stackrel{24}{=}$, we will assign all cluster structures to their structural type families and their symmetry point groups.

A new class of structures which introduces a new region in the structural phase diagram 24,25 for large system sizes $N \geq 98$ and $\sigma \geq 1.25$ is the global minimum structure of $B L J_{100, \sigma=1.3}$. The polytetrahedral structure with disclination network can be classified as $4 Z 14$ structure with point group symmetry $C_{S}$. Generally, $Z 14$-atoms are part of a single disclination line whereas $Z 15$ (see Fig and $Z 16$ atoms act as nodes connecting 3 or 4 disclination lines respectively. Disclination lines always pass edges with six tetrahedra around them, see Fig [5

The 4 atoms with coordination number $Z=14$ form 4 pairwise disconnected (single) disclination lines ending at $4 Z=13$ shell-atoms 28 . This type of structure is also the corresponding putative global minimum structure of $B L J_{99, \sigma=1.3}, B L J_{100, \sigma=1.25}, B L J_{99, \sigma=1.25}$ and $B L J_{98, \sigma=1.25}$. The core of these structures consists of 42 atoms and is completely covered by the shell atoms (pure core-shell). Depending on the cluster size there is only an absence of one or two type-B shell atoms. The putative ground state energy of $B L J_{100, \sigma=1.3}$ is -604.796307 in common units.

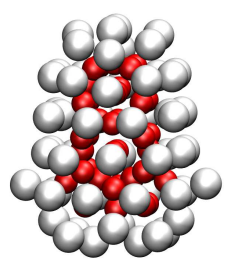

(a)

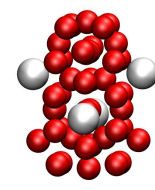

(b)

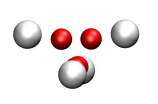

(c)
FIG. 4. $4 Z 14$ structure of $B L J_{100, \sigma=1.3}$ with $C_{S}$ symmetry and the putative ground state energy -604.796307. a) Shows the whole cluster. b) Disclination network embedded into the core. c) Disclination network

A summary of the structural types and energies corresponding to all other minima we found is given in table [II] Fig. [5 shows the $A_{39} B_{58}$ composition of $B L J_{97, \sigma=1.25}$ and how the disclinations are embedded into the whole cluster. It is a polytetrahedral $Z 15$ structure with $C_{1}$ symmetry and the typical $Z 15$ disclination network. The putative global minimum energy is -578.201634 .

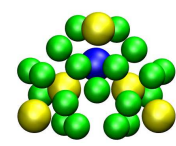

(a)

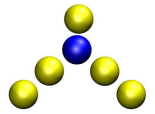

(b)

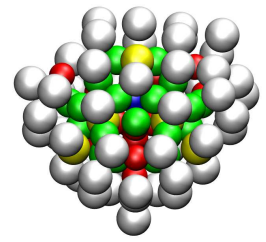

(c)
FIG. 5. Putative global minimum structure of $B L J_{97, \sigma=1.25}$ represented by the $A_{39} B_{58}$ composition. a) 3 (single) disclination lines (yellow) passing edges with 6 tetrahedra around them (green), connected at the $Z 15$-node (blue). b) Disclination network coloured. c) Shows the whole cluster in corresponding colours

Doye et al $l^{24}$ describe the structural motives of other common binary Lennard-Jones structures up to a system size $N=100$.

\section{CONCLUSIONS}

Molecular dynamics based moves were found to be optimal in the context of global optimization. According to the Bell Evans Polyani principle one should escape over low barriers. Even though moves that escape over the lowest saddle point around a local minima are consequently expected to perform better, if the number of visited minima is taken as the success criterion, it turns out that molecular dynamics based moves are more efficient for energy landscapes which have a strong funnel like structure, since in this case the MD trajectory can cross over several saddle points. For other energy land- 


\begin{tabular}{llcccc}
\hline$N$ & $\sigma$ & $\epsilon$ & $\begin{array}{c}\text { stoichio- } \\
\text { metry }\end{array}$ & $\begin{array}{c}\text { point } \\
\text { group }\end{array}$ & $\begin{array}{c}\text { structural } \\
\text { type }\end{array}$ \\
\hline 100 & 1.3 & -604.796307 & $A_{42} B_{58}$ & $C_{s}$ & $4 \mathrm{Z} 14$ \\
99 & 1.3 & -597.592233 & $A_{42} B_{57}$ & $C_{s}$ & $4 \mathrm{Z} 14$ \\
98 & 1.3 & -590.787413 & $A_{41} B_{57}$ & $C_{1}$ & $\mathrm{Z} 14$ \\
97 & 1.3 & -583.871531 & $A_{41} B_{56}$ & $C_{1}$ & $\mathrm{Z} 14$ \\
96 & 1.3 & -576.517002 & $A_{41} B_{55}$ & $C_{1}$ & $\mathrm{Z} 14$ \\
100 & 1.25 & -599.264624 & $A_{42} B_{58}$ & $C_{s}$ & $4 \mathrm{Z} 14$ \\
99 & 1.25 & -592.138846 & $A_{42} B_{57}$ & $C_{s}$ & $4 \mathrm{Z} 14$ \\
98 & 1.25 & -584.930661 & $A_{42} B_{56}$ & $C_{s}$ & $4 \mathrm{Z} 14$ \\
97 & 1.25 & -578.201634 & $A_{39} B_{58}$ & $C_{1}$ & $\mathrm{Z} 15$ \\
96 & 1.25 & -571.389275 & $A_{39} B_{57}$ & $C_{1}$ & $\mathrm{Z} 15$ \\
100 & 1.2 & -591.768143 & $A_{35} B_{65}$ & $C_{1}$ & $\mathrm{Z} 16 \mathrm{Z} 15$ \\
97 & 1.2 & -571.392434 & $A_{33} B_{64}$ & $C_{1}$ & $\mathrm{Z} 16 \mathrm{Z} 15$ \\
96 & 1.2 & -564.674461 & $A_{33} B_{63}$ & $C_{1}$ & $\mathrm{Z} 16 \mathrm{Z15}$ \\
95 & 1.2 & -557.690639 & $A_{33} B_{62}$ & $C_{1}$ & $\mathrm{Z} 16 Z 15$ \\
94 & 1.15 & -542.476905 & $A_{31} B_{63}$ & $C_{1}$ & $\mathrm{Z} 16 Z 15$ \\
93 & 1.15 & -535.594853 & $A_{31} B_{62}$ & $C_{1}$ & $\mathrm{Z} 16 Z 15$ \\
92 & 1.15 & -529.190149 & $A_{28} B_{64}$ & $C_{1}$ & $\mathrm{Z} 16 Z 15$ \\
\hline
\end{tabular}

TABLE III. System size $N$, energies $\epsilon$, compositions, point groups and structural types of the new putative global minima.

scapes MD based escapes are about equally efficient in terms of the number of distinct visited minima, but are more efficient in terms of the total number of visited minima, i.e. in terms of the number of local geometry optimizations. In practice moves that escape over the lowest saddle point are too expensive since they require a complete exploration of the potential energy surface around the local minimum from which one wants to escape. In practice the only saddle point escape moves that are affordable would be moves where one searches for a single saddle point in a soft direction. It turns out that these saddle points can sometimes be very high and the approach is therefore much less efficient that an approach using molecular dynamics based moves in soft directions. The important difference is that by energy conservation the MD based trajectory can not cross over energetically high saddle points whereas saddle point searches in soft directions can give very high saddle points. In addition MD based escapes require significantly less force evaluations (of the order of 100) than even a single saddle point search. The value of the parameter $E_{\text {diff }}$ in the Minima Hopping method is a good measure of the quality of the moves. If moves lead on average in other low energy configurations $E_{\text {diff }}$ will be small and one has to search only over low energy structures. One has therefore to search only over a number of local minima which is much smaller than in the case where one has to search in a larger energy window above the global minimum. According to this criterion identity exchange moves are in general worse than MD based moves except for very small values of $\sigma$. If identity exchange moves are however added as some kind of post processing step to a MD based move without the need of an additional geometry optimization for each exchange trial, the efficiency of the Minima Hopping method can be improved. With such an improved version of the Minima Hopping method we were able to find several new global minima structures for binary Lennard Jones clusters with up to 100 atoms and size ratios of $\sigma=1.15$. For large values of $\sigma$ the ordinary Minima Hopping method without identity exchanges was used and turned out to be powerful enough to find new global minima structures for size rations of $\sigma=1.2,1.25$ and 1.3 .

Financial support from SNF and computing time from CSCS are acknowledged. We gratefully acknowledge expert discussions with Riccardo Ferrando.

\section{REFERENCES}

${ }^{1}$ Riccardo Ferrando, Alessandro Fortunelli and Roy L. Johnston, Phys.Chem.Chem.Phys, 2008,10, 640-649

${ }^{2}$ G.Rossi and R.Ferrando, J.Phys.:Condens. Matter 21(2009)

${ }^{3}$ M. A. Neumann, J. Phys. Chem. B 1129810 (2008)

${ }^{4}$ A.J. Morris, C.J. Pickard, and R.J. Needs Phys. Rev. B 80, 144112 (2009)

${ }^{5}$ Z. Li and H. A. Scheraga, Proc. Natl. Acad. Sci. USA, 846611 (1987)

${ }^{6}$ Kirkpatrick, S.; C. D. Gelatt, M. P. Vecchi, Science 220671 (1983)

${ }^{7}$ S. Goedecker, J. Chem. Phys. 120, 9911 (2004).

${ }^{8}$ D. A. Richie, J. Kim, S. A. Barr, K. R. A. Hazzard, R. G. Hennig, and J. W. Wilkins. Physical Review Letters 92, 45501 (2004)

${ }^{9}$ J. Rogan, G. Garca, C. Loyola, W. Orellana, R. Ramrez, and M. Kiwi J. Chem. Phys. 125, 214708 (2006)

${ }^{10}$ Michael C. Prentiss, David J. Wales, and Peter G. Wolynes J. Chem. Phys. 128, 225106 (2008)

${ }^{11}$ Normand Mousseau and G. T. Barkema Phys. Rev. E 57, 2419 (1998)

12 J. Chem. Phys. 112, 9599 (2000); doi:10.1063/1.481576 (8 pages) Temperature-accelerated dynamics for simulation of infrequent events Mads R. Srensen and Arthur F. Voter

${ }^{13}$ Hartke, B., J. of Comp. Chem. 201752 (1999)

${ }^{14}$ Deaven, D.M., Ho, K.M., Physical Review Letters 75 288 (1995)

${ }^{15}$ Oganov A.R., Glass C.W., J. Chem. Phys. 124244704 (2006)

${ }^{16}$ S. Roy, S. Goedecker, and V. Hellmann, Phys. Rev. E 77, 056707 (2008).

${ }^{17} \mathrm{~F}$. Jensen, Introduction to Computational Chemistry, Wiley, New York (1999).

18 http://www-wales.ch.cam.ac.uk/CCD.html

${ }^{19}$ Francesca Balleto and Riccardo Ferrando, Review of modern physics, $\mathbf{7 7},(2005)$

${ }^{20}$ G. Henkelman and H. Jónsson, J. Chem. Phys. 111, 7010 (1999)

${ }^{21}$ P. Pulay, Chem. Phys. Lett., 73, 393, (1980)

${ }^{22}$ Sandro Schoenborn, Stefan Goedecker, Shantanu Roy and Artem Oganov, J. Chem. Phys. 130144108 (2009) 
${ }^{23}$ J. Jellinek and E. B. Krissinel, Chem. Phys. Let. 258 283 (1996)

${ }^{24}$ Jonathan P. K. Doye and Lars Meyer, The structure of binary Lennard-Jones clusters: The effects of atomic size ratio (2006)

${ }^{25}$ Jonathan P. K. Doye and Lars Meyer, Phys. Rev. Lett, 95, 063401, (2005)
${ }^{26}$ Cassioli Andrea and Locatelli Marco and Schoen Fabio, Optimization Methods and Software 24, 4-5,(2009)

${ }^{27}$ W.J.Pullan, J, of Comp. Chem., 18, 8, 1997.

${ }^{28}$ Atoms with coordination number $Z=13$ often act as ending points of disclination lines in a truncated edge 